\title{
Error of the Newton-Cotes and Gauss-Legendre Quadrature Formulas
}

\author{
By N. S. Kambo
}

\begin{abstract}
It was shown by P. J. Davis that the Newton-Cotes quadrature formula is convergent if the integrand is an analytic function that is regular in a sufficiently large region of the complex plane containing the interval of integration. In the present paper, a bound on the error of the Newton-Cotes quadrature formula for analytic functions is derived. Also the bounds on the Legendre polynomial and the Legendre function of the second kind are obtained. These bounds are employed to derive a bound on the error of the Gauss-Legendre quadrature formula for analytic functions.
\end{abstract}

1. Introduction. Let $z_{0}, z_{1}, \ldots, z_{n-1}$ be $n$ distinct points lying in the complex plane and let $C$ be a closed contour containing these points in its interior. Suppose that $f(z)$ is a regular function within $C$. Let

$$
w_{n}(z)=\prod_{i=0}^{n-1}\left(z-z_{i}\right) .
$$

If now the points $z=a$ and $z=b$ also lie inside $C$, then, following the method given in [1, pp. 117-118], we have for any weight function $g(t)$,

$$
\int_{a}^{b} g(t) f(t) d t=\sum_{k=0}^{n-1} \lambda_{k} f\left(z_{k}\right)+E_{n}(f)
$$

where $\lambda_{0}, \lambda_{1}, \ldots, \lambda_{n-1}$ are the weights for interpolating integration at $z_{0}, z_{1}, \ldots, z_{n-1}$ and

$$
\begin{gathered}
E_{n}(f)=\frac{1}{\pi i} \int_{c} \frac{f(z) v_{n}(z) d z}{w_{n}(z)}, \\
v_{n}(z)=\frac{1}{2} \int_{a}^{b} \frac{w_{n}(t) g(t) d t}{z-t},
\end{gathered}
$$

and

$$
\lambda_{k}=\int_{a}^{b} \frac{g(t) w_{n}(t) d t}{\left(t-z_{k}\right) w_{n}^{\prime}\left(z_{k}\right)} \quad(k=0,1, \ldots, n-1) .
$$

Here $E_{n}(f)$ designates the error of the $n$-point interpolating quadrature formula given by

Received August 11, 1969, revised November 7, 1969.

AMS Subject Classifications. Primary 6555, 6580; Secondary 3340.

Key Words and Phrases. Newton-Cotes quadrature formula, Cotes numbers, Legendre polynomial, Legendre function of the second kind, Gauss-Legendre quadrature formula, error bound, asymptotic bound, Davis' method. 


$$
\int_{a}^{b} g(t) f(t) d t \approx \sum_{k=0}^{n-1} \lambda_{k} f\left(z_{k}\right)
$$

The problem of estimating the error $E_{n}(f)$ for analytic functions is connected with the estimation of $v_{n}(z)$ and $w_{n}(z)$ on $C$. In this paper, we shall bound the error of two special quadrature formulas over the interval $[-1,1]$ (i.e., $a=-1, b=+1$ ) with the weight function $g(t)=1$. For the contour $C$ we shall take the ellipse $E_{\rho}$ in the complex plane defined by

$$
z=\frac{1}{2}\left(\xi+\xi^{-1}\right), \quad \xi=\rho e^{i \theta}, \quad 0 \leqq \theta \leqq 2 \pi \text { and } \rho>1 .
$$

The ellipse $E_{\rho}$ has foci at $z= \pm 1$ and sum of its semi-axes is $\rho(>1)$. Let

$$
A\left(E_{\rho}\right)=\left\{f: \begin{array}{l}
f \text { is analytic on }[-1,1] \text { and continuable analytically so as to be } \\
\text { single valued and regular in the closure of the ellipse } E_{\rho} .
\end{array}\right.
$$

Davis [2] discussed the convergence (as $n \rightarrow \infty$ ) of the Newton-Cotes quadrature formula for functions $f \in A\left(E_{\rho}\right)$. In fact, it was shown by him that the Newton-Cotes quadrature formula is convergent provided $f \in A\left(E_{\rho}\right)$, where $\rho>2$. Moreover, as $n \rightarrow \infty$, the error $E_{n}(f)$ tends to zero with geometric rapidity. In Section 2 we derive an upper bound on the error $\left|E_{N C_{n}}(f)\right|$ of the Newton-Cotes quadrature formula given in Theorem 1. For $\rho>1+\sqrt{ } 2$, this bound on $\left|E_{N C_{n}}(f)\right|$ converges $($ as $n \rightarrow \infty)$ to zero with geometric rapidity.

In Section 3 we derive an upper bound on $\left|Q_{n}(z)\right|$ and a lower bound on $\left|P_{n}(z)\right|$ for $z \in E_{\rho}$ and $\rho>\sqrt{ } 2$, where $P_{n}(z)$ and $Q_{n}(z)$ are the Legendre polynomial and Legendre function of the second kind, respectively. Using these bounds, we derive an upper bound on the error $\left|E_{G_{n}}(f)\right|$ of the Gauss-Legendre quadrature formula given in Theorem 2. This result differs from similar results due to Chawla and Jain, since this result is a bound, while theirs is an asymptotic bound, i.e., a bound on the first term of the asymptotic expansion.

2. Error of the Newton-Cotes Quadrature Formula. Newton-Cotes $(n+1)$-point quadrature formula for the integral $\int_{-1}^{+1} f(t) d t$ is a special case of the nonsymmetric interpolating quadrature formula (2) when $a=-1, b=+1, g(t)=1$, and the abscissas are given by

$$
z_{i}=x_{i}=-1+2 i / n \quad(i=0,1, \ldots, n) .
$$

The weights $\lambda_{k}$ (known as Cotes numbers) and the error $E_{N C_{n}}(f)$ of the Newton-Cotes quadrature formula are, respectively, given by

$$
\lambda_{k}=\int_{-1}^{+1} \frac{w_{n+1}(x) d x}{\left(x-x_{k}\right) w_{n+1}^{\prime}\left(x_{k}\right)} \quad(k=0,1, \ldots, n)
$$

and

where

$$
E_{N C_{n}}(f)=\frac{1}{\pi i} \int_{C} \frac{f(z) Q_{n}^{*}(z) d z}{w_{n+1}(z)}
$$

$$
Q_{n}^{*}(z) \equiv v_{n+1}(z)=\frac{1}{2} \int_{-1}^{+1} \frac{w_{n+1}(x) d x}{z-x}
$$


and $w_{n+1}(x)$ is defined by (1), with $z_{i}$ given by (8).

2.1 An Upper Bound on $Q_{n}^{*}(z)$.

Lemma 1. For $z \in E_{\rho}$,

$$
Q_{n}^{*}(z)=\sum_{m=1}^{\infty} \frac{\sigma_{n m}^{*}}{\xi m}
$$

where

$$
\sigma_{n m}^{*}=\int_{0}^{\pi} w_{n+1}(\cos \theta) \sin m \theta d \theta, \quad(n, m=1,2,3, \ldots)
$$

and $\sigma_{n m}^{*}$ is bounded as follows:

(a) Let $n \geqq 1$ be an odd integer. Then

$$
\begin{aligned}
\left|\sigma_{n m}^{*}\right| & \leqq 0, \quad m \text { even } \\
& \leqq 4 / 3, \quad m=1, \\
& \leqq \sqrt{ } 2(\pi / 4), \quad m>1,
\end{aligned}
$$

and

$$
\left|Q_{n}^{*}(z)\right| \leqq 4 \rho / 3\left(\rho^{2}-1\right) .
$$

(b) Let $n \geqq 1$ be an even integer. Then

$$
\begin{aligned}
\left|\sigma_{n m}^{*}\right| & \leqq 0, \quad m \text { odd } \\
& \leqq 2\left[\frac{m^{2}}{18}\left(\frac{3}{4 m^{2}-1}+\frac{1}{4 m^{2}-9}\right)\right]^{1 / 2} \leqq 4\left(\frac{2}{105}\right)^{1 / 2}, \quad m \geqq 2
\end{aligned}
$$

and

$$
\left|Q_{n}^{*}(z)\right| \leqq 4 \sqrt{ }(2 / 105)\left(1 /\left(\rho^{2}-1\right)\right) .
$$

Proof. In (11), setting $x=\cos \theta$ and $z=\frac{1}{2}\left(\zeta+\zeta^{-1}\right)$, we get

$$
Q_{n}^{*}(z)=\frac{1}{\xi} \int_{0}^{\pi} \frac{w_{n+1}(\cos \theta) \sin \theta d \theta}{1-2 \xi^{-1} \cos \theta+\xi^{-2}} .
$$

Following [3, pp. 311-312], we get

$$
\frac{\sin \theta}{\xi}\left[1-2 \xi^{-1} \cos \theta+\xi^{-2}\right]^{-1}=\sum_{m=1}^{\infty} \frac{\sin m \theta}{\xi m} \text {. }
$$

The last series converges uniformly and absolutely for $0 \leqq \theta \leqq \pi$, and for all $|\xi| \geqq \rho>1$. Using (19) in (18), we obtain (12). We now proceed to find bounds for

$$
\sigma_{n m}^{*}=\int_{0}^{\pi}\left[\prod_{i=0}^{n}\left(\cos \theta-x_{i}\right)\right] \sin m \theta d \theta .
$$

Proof of (a). Let $n$ be odd, say, $n=2 r-1(r=1,2,3, \ldots)$. From (20)

$$
\sigma_{n m}^{*}=\int_{0}^{\pi}\left[\prod_{i=0}^{r-1}\left(\cos ^{2} \theta-x_{i}^{2}\right)\right] \sin m \theta d \theta .
$$


Breaking the range of integration into two intervals, $[0, \pi / 2]$ and $[\pi / 2, \pi]$, we easily get

$$
\sigma_{n m}^{*}=\left[1+(-1)^{m+1}\right] \int_{0}^{\pi / 2}\left[\prod_{i=0}^{r-1}\left(\cos ^{2} \theta-x_{i}^{2}\right)\right] \sin m \theta d \theta .
$$

Hence $\sigma_{n m}^{*}=0$ if $m$ is even. We now bound $\sigma_{n m}^{*}$ for odd integer $m$. Consider the following two cases.

Case 1. Let $m=1$. From (21)

$$
\left|\sigma_{n m}^{*}\right| \leqq 2 \int_{0}^{\pi / 2} \sin ^{3} \theta d \theta=4 / 3 .
$$

Case 2. Let $m>1$. (21) yields

$$
\begin{aligned}
\left|\sigma_{n m}^{*}\right| & \leqq 2 \int_{0}^{\pi / 2} \sin ^{2} \theta|\sin m \theta| d \theta \\
& \leqq 2\left[\left(\int_{0}^{\pi / 2} \sin ^{2} \theta d \theta\right)\left(\int_{0}^{\pi / 2} \sin ^{2} \theta \sin ^{2} m \theta d \theta\right)\right]^{1 / 2} \\
& =\sqrt{ } 2(\pi / 4)
\end{aligned}
$$

where the second inequality is derived by employing Cauchy's inequality. This completes the proof of (14).

Proof of (b). Let $n$ be an even integer, say, $n=2 r(r=1,2,3, \ldots)$. We have from (20),

$$
\begin{aligned}
\sigma_{n m}^{*} & =\int_{0}^{\pi}\left[\prod_{i=0 ; i \neq r}^{2 r}\left(\cos \theta-x_{i}\right)\right] \cos \theta \sin m \theta d \theta \\
& =\int_{0}^{\pi}\left[\prod_{i=0}^{r-1}\left(\cos ^{2} \theta-x_{i}^{2}\right)\right] \cos \theta \sin m \theta d \theta \\
& =\left[1+(-1)^{m+2}\right] \int_{0}^{\pi / 2}\left[\prod_{i=0}^{r-1}\left(\cos ^{2} \theta-x_{i}^{2}\right)\right] \cos \theta \sin m \theta d \theta .
\end{aligned}
$$

Hence $\sigma_{n m}^{*}=0$ for $m$ odd. When $m$ is even, we obtain

$$
\begin{aligned}
\left|\sigma_{n m}^{*}\right| & \leqq 2 \int_{0}^{\pi / 2} \sin ^{2} \theta \cos \theta|\sin m \theta| d \theta \\
& \leqq 2\left[\left(\int_{0}^{\pi / 2} \sin ^{2} \theta \cos \theta d \theta\right)\left(\int_{0}^{\pi / 2} \sin ^{2} \theta \sin ^{2} m \theta \cos \theta d \theta\right)\right]^{1 / 2} \\
& =2\left[\frac{m^{2}}{18}\left(\frac{3}{4 m^{2}-1}+\frac{1}{4 m^{2}-9}\right)\right]^{1 / 2} \leqq 4 \sqrt{ }(2 / 105)
\end{aligned}
$$

This proves (16). Bounds (15) and (17) follow from (12), (14), and (16). This completes the proof of Lemma 1.

2.2 A Lower Bound on $w_{n+1}(z)$.

Lemma 2. For $z \in E_{\rho}$,

$$
\left|w_{n+1}(z)\right| \geqq\left(\left(\rho^{2}-1\right) /(2 \rho)\right)^{n+1} .
$$

Proof. We consider the two cases when $n$ is odd or even. 
Case 1. When $n=2 r-1(r=1,2,3, \ldots)$ is odd, we have for $z \in E_{\rho}$,

$$
\begin{aligned}
\left|w_{n+1}(z)\right| & =\frac{1}{(2 \rho)^{n+1}} \prod_{i=0}^{r-1}\left|\xi^{4}+1+2 \xi^{2}-4 \xi^{2} x_{i}^{2}\right| \\
& =\frac{1}{(2 \rho)^{n+1}} \prod_{i=0}^{r-1}\left|\left(\xi^{2}-q_{i}\right)\left(\xi^{2}-\bar{q}_{i}\right)\right|,
\end{aligned}
$$

where $q_{i}=\left(2 x_{i}^{2}-1\right)+\sqrt{ }-1\left(2 x_{i}\left(1-x_{i}^{2}\right)^{1 / 2}\right)$ and $\bar{q}_{i}$ is the complex conjugate of $q_{i}$. Also $\left|q_{i}\right|=1$. Hence,

$$
\left|w_{n+1}(z)\right| \geqq \frac{1}{(2 \rho)^{n+1}} \prod_{i=0}^{r-1}\left(\rho^{2}-1\right)^{2}=\left(\frac{\rho^{2}-1}{2 \rho}\right)^{n+1} .
$$

Case 2. When $n=2 r(r=1,2,3, \ldots)$ is even, we have for $z \in E_{\rho}$,

$$
\begin{aligned}
\left|w_{n+1}(z)\right| & =|z| \prod_{i=0}^{r-1}\left|z^{2}-x_{i}^{2}\right| \\
& =\frac{1}{2|\xi|}\left|\xi^{2}+1\right|\left(\frac{1}{2|\xi|}\right)^{2 r} \prod_{i=0}^{r-1}\left|\left(\xi^{2}-q_{i}\right)\left(\xi^{2}-\bar{q}_{i}\right)\right| \\
& \geqq\left(\rho^{2}-1\right) /(2 \rho)^{n+1}\left(\rho^{2}-1\right)^{2 r}=\left(\left(\rho^{2}-1\right) / 2 \rho\right)^{n+1} .
\end{aligned}
$$

This completes the proof of Lemma 2.

$2.3 A$ Bound on $E_{N C_{n}}(f)$. From (10), by selecting the contour $C$ as an ellipse $E_{\rho}$ $(\rho>1)$ it follows that

$$
\left|E_{N C_{n}}(f)\right| \leqq \frac{1}{\pi} \int_{E_{\rho}} \frac{|f(z)|\left|Q_{n}^{*}(z)\right|}{\left|w_{n+1}(z)\right|}|d z| .
$$

Employing (15), (17), and (22) we obtain the following theorem.

IIII.)RIM 1. Let $f \in A\left(E_{\rho}\right), \rho>1$. Then for $n \geqq 1$

$$
\left|E_{N C_{n}}(f)\right| \leqq K_{\rho} M(\rho)\left(2 \rho /\left(\rho^{2}-1\right)\right)^{n+1},
$$

where

$$
\begin{aligned}
K_{\rho} & =\frac{4}{3} \frac{\left(\rho^{2}+1\right)}{\left(\rho^{2}-1\right)}, \quad n \text { odd }, \\
& =4\left(\frac{2}{105}\right)^{1 / 2}\left(\frac{\rho^{2}+1}{\rho\left(\rho^{2}-1\right)}\right), \quad \text { n even },
\end{aligned}
$$

and

$$
M(\rho)=\max |f(z)| \quad \text { on } E_{\rho} .
$$

2.4 Discussion of the Results. Relation (24) gives a bound on $\left|E_{N C_{n}}(f)\right|$ for all $\rho>1$ and all $n \geqq 1$. From (24) it is easily seen that the bound on $\left|E_{N C_{n}}(f)\right|$ tends to zero as $n$ tends to infinity only if $\rho>1+\sqrt{ } 2$. However, Davis [2] proved the stronger result that $\left|E_{N C_{n}}(f)\right|$ tends to zero as $n$ tends to infinity provided that $\rho>2$. Therefore, it is of interest to tighten the bound (24).

3. Error of the Gauss-Legendre Quadrature Formula. An important special case of the general interpolating quadrature formula (2) is the Gauss-Legendre quadrature 
formula. The $n$-point Gauss-Legendre quadrature formula for $\int_{-1}^{+1} f(t) d t$ is obtained by letting $a=-1, b=+1, g(t)=1$, and choosing $z_{0}, z_{1}, z_{2}, \ldots, z_{n-1}$ as the $n$ zeros of the Legendre polynomial $P_{n}(x)$. The weights $\lambda_{k}$ and the error $E_{G_{n}}(f)$ of the Gauss-Legendre quadrature formula are, respectively, given by

$$
\lambda_{k}=\int_{-1}^{+1} \frac{P_{n}(x) d x}{\left(x-z_{k}\right) P_{n}^{\prime}\left(z_{k}\right)} \quad(k=0,1, \ldots, n-1)
$$

and

$$
E_{G_{n}}(f)=\frac{1}{\pi i} \int_{C} \frac{f(z) Q_{n}(z) d z}{P_{n}(z)},
$$

where $Q_{n}(z)$, defined by

$$
Q_{n}(z)=\frac{1}{2} \int_{-1}^{+1} \frac{P_{n}(t) d t}{z-t},
$$

is the Legendre function of the second kind.

3.1 An Upper Bound on $Q_{n}(z)$.

Lemma 3. For $z \in E_{\rho}$

$$
Q_{n}(z)=\frac{1}{\xi^{n+1}} \sum_{r=0}^{\infty} \frac{\sigma_{n, n+2 r+1}}{\xi^{2 r}},
$$

where

$$
0<\sigma_{n, n+2 r+1} \leqq b_{n} \text { for } \quad r=0,1,2,3, \ldots
$$

Moreover,

$$
\left|Q_{n}(z)\right| \leqq \rho b_{n} /\left(\rho^{2}-1\right) \rho^{n},
$$

where

$$
b_{n}=(2)^{2 n+1}(n !)^{2} /(2 n+1) ! \quad(\leqq 2 \text { for all } n=0,1,2, \ldots) .
$$

Proof. Setting $t=\cos \theta$ and $z=\frac{1}{2}\left(\xi+\xi^{-1}\right)$ in (29) and proceeding as in the proof of Lemma 1, we get

$$
Q_{n}(z)=\sum_{m=1}^{\infty} \frac{\sigma_{n m}}{\xi^{m}}
$$

where

$$
\sigma_{n m}=\int_{0}^{\pi} P_{n}(\cos \theta) \sin (m \theta) d \theta .
$$

Heine [4, p. 311] gave the following evaluation of $\sigma_{n m}$ :

$$
\sigma_{n m}=\Gamma\left(\frac{m+n+1}{2}\right) \Gamma\left(\frac{m-n}{2}\right) / \Gamma\left(\frac{m-n+1}{2}\right) \Gamma\left(\frac{m+n+2}{2}\right),
$$

$=0$, otherwise. 
Writing $m-n=2 r+1(r=0,1,2, \ldots)$, we obtain from (36)

$$
\frac{\sigma_{n, n+2 r+1}}{\sigma_{n, n+2 r+3}}=\frac{(r+1)(n+r+3 / 2)}{(r+1 / 2)(n+r+1)}>1 .
$$

Hence, for fixed $n$, the sequence $\left\{\sigma_{n, n+2 r+1}\right\}$ for varying $r$ is strictly monotonically decreasing, yielding

$$
\sigma_{n, n+2 r+1}<\sigma_{n, n+1}=b_{n}=\frac{(n !)^{2}(2)^{2 n+1}}{(2 n+1) !} .
$$

The results (30) and (31) follow from (34), (36), and (37). Bound (32) follows from (30) and (31). This completes the proof of the lemma.

A similar result based on $\left|\sigma_{n, k}\right| \leqq 2$ was used to derive an asymptotic bound $(n \rightarrow \infty)$ on $\left|E_{G_{n}}(f)\right|$ (see [5]). Using Stirling's formula

$$
b_{n} \approx 2 \sqrt{ } \pi n /(2 n+1) \text { as } n \rightarrow \infty .
$$

This extra $n^{-1 / 2}$ factor in (32) is vital to obtain a better bound.

3.2 A Lower Bound on $P_{n}(z)$.

LIMMA 4. For $z \in E_{\rho}$,

$$
\begin{aligned}
\left|P_{n}(z)\right| & \geqq a_{n}\left(\rho^{2}+\rho^{-2 n}-2\right) \rho^{n} /\left(\rho^{2}-1\right) \\
& \geqq a_{n}\left(\rho^{2}-2\right) \rho^{n} /\left(\rho^{2}-1\right),
\end{aligned}
$$

where

$$
a_{n}=\frac{(2 n) !}{(2)^{2 n}(n !)^{2}}=\frac{2}{(2 n+1) b_{n}} \quad\left(\approx \frac{1}{\sqrt{ } \pi n} \text { as } n \rightarrow \infty\right) .
$$

The bound (39) is significant if $\rho>\sqrt{ } 2$.

Proof. It is shown in [3, Lemmas 12.4.1 and 12.4.3] that for $z \in E_{\rho}$

$$
P_{n}(z)=\sum_{j=0}^{n} a_{j} a_{n-j}(\xi)^{n-2 j}
$$

and

$$
a_{j} a_{n-j} \leqq a_{n}, \quad j=0,1,2, \ldots, n,
$$

where $a_{n}$ is given by (40). From (41)

$$
\begin{aligned}
\left|P_{n}(z)\right| & =\left|1+\sum_{j=1}^{n} \frac{a_{j} a_{n-j}}{a_{n}} \xi^{-2 j}\right|\left(a_{n} \rho^{n}\right) \\
& \geqq a_{n} \rho^{n}\left[1-\left|\sum_{j=1}^{n} \frac{a_{j} a_{n-j}}{a_{n}} \xi^{-2 j}\right|\right] \\
& \geqq a_{n} \rho^{n}\left[1-\sum_{j=1}^{n} \rho^{-2 j}\right] \quad(\operatorname{using}(42)) \\
& =a_{n} \rho^{n}\left[\frac{\rho^{2}+\rho^{-2 n}-2}{\rho^{2}-1}\right] \geqq a_{n} \rho^{n} \frac{\left(\rho^{2}-2\right)}{\left(\rho^{2}-1\right)} .
\end{aligned}
$$

This establishes the lemma. 
$3.3 A$ Bound on $E_{G_{n}}(f)$. From (27) by selecting the contour $C$ as an ellipse $E_{\rho}$ it follows that

$$
\left|E_{G_{n}}(f)\right| \leqq \frac{1}{\pi} \int_{E_{\rho}} \frac{|f(z)|\left|Q_{n}(z)\right||d z|}{\left|P_{n}(z)\right|} .
$$

Let $\rho>\sqrt{ } 2$. Employing (32) and (39), we obtain

$$
\left|E_{G_{n}}(f)\right| \leqq d_{n} \frac{M(\rho)}{\rho^{2 n}}\left(\frac{\rho^{2}+1}{\rho^{2}-2}\right)
$$

where

$$
d_{n}=\frac{(2)^{4 n+1}(n !)^{4}}{(2 n) !(2 n+1) !}
$$

and $M(\rho)$ is given by (26).

It is easily seen that the sequence $\left\{d_{n}\right\}$ is strictly monotonically increasing and $\lim _{n \rightarrow \infty} d_{n}=\pi$. Thus

$$
0<d_{n} \leqq \pi
$$

and we have the following theorem.

THI: ()REM 2. Let $f \subseteq A\left(E_{\rho}\right), \rho>\sqrt{ } 2$. Then

$$
\left|E_{G_{n}}(f)\right| \leqq d_{n} \frac{\left(\rho^{2}+1\right)}{\left(\rho^{2}-2\right)} \frac{M(\rho)}{\rho^{2 n}},
$$

where $M(\rho)$ and $d_{n}$ are, respectively, given by (26) and (45).

In view of (46), an overall bound is given by

CoR(II.ARY. Let $n \rightarrow \infty$, then for $\rho>\sqrt{ } 2$

$$
\left|E_{G_{n}}(f)\right| \leqq \pi \frac{\left(\rho^{2}+1\right)}{\left(\rho^{2}-2\right)} \frac{M(\rho)}{\rho^{2 n}} .
$$

We mention here that Chawla [6, Theorem 1] obtained the following asymptotic $(n \rightarrow \infty)$ result by using Davis' method.

If $f \in A\left(E_{\rho}\right)$ and $\rho>1$, then

$$
\left|E_{G_{n}}(f)\right| \leqq \pi \frac{M(\rho)}{\rho^{2 n}}\left(1+\rho^{-4}\right)^{1 / 2}(1+O(1)) .
$$

3.4 Discussion of the Results. The asymptotic $(n \rightarrow \infty)$ bound $(48)$ is not better than the asymptotic bound (49). But the bound (47) is valid for all $n$ and $\rho>\sqrt{ } 2$ as compared to the asymptotic bound (49) which is valid for $n \rightarrow \infty$ and $\rho>1$.

As a consequence of (47) we have the following asymptotic bound valid for $\rho \rightarrow \infty$

$$
\left|E_{G_{n}}(f)\right| \leqq d_{n} \frac{M(\rho)}{\rho^{2 n}}\left[1+\frac{3}{\rho^{2}-2}\right]=d_{n} \frac{M(\rho)}{\rho^{2 n}}\left[1+O\left(\rho^{-2}\right)\right] .
$$

This bound is compatible with the asymptotic $(\rho \rightarrow \infty)$ bound of Chawla (unpublished work), having the same leading term.

The bound (47) can be compared in some sense with the bound of Stenger [7] given by 


$$
\left|E_{G_{n}}(f)\right| \leqq \frac{32}{\pi} \frac{M_{1}(\rho)}{\rho^{2 n}},
$$

where $M_{1}(\rho)=\max _{z \in E_{\rho}} \mid$ Real part of $f(z) \mid$ and $\rho>1$. Now, if we compare the coefficients $\left(\left(\rho^{2}+1\right) /\left(\rho^{2}-2\right)\right) d_{n}$ and $32 / \pi$ of $(47)$ and (51), respectively, it is clear that the former is less than the latter, for

$$
\rho>\left(\frac{64+\pi d_{n}}{32-\pi d_{n}}\right)^{1 / 2} .
$$

The relation (52) is definitely true if

$$
\rho>\left(\frac{64+\pi^{2}}{32-\pi^{2}}\right)^{1 / 2} \quad(\approx 1.83) .
$$

Thus we see that Stenger's bound is better for small $\rho$, but ours is better for large $\rho$. For small $\rho<\sqrt{ } 2$ ours does not exist. For large $\rho$ ours is smaller by a factor $\pi^{2} / 32=$ 0.31 . The cross-over point is near $\rho=1.83$. These numbers depend on $n$ but only slightly.

Indian Institute of Technology

Haus Khas, New Delhi-29, India

1. P. J. Davis \& P. Rabinowitz, Numerical Integration, Blaisdell, Waltham, Mass., 1967. MR 35 \#2482.

2. P. J. Davis, "On a problem in the theory of mechanical quadratures," Pacific J. Math., v. 5, 1955, pp. 669-674. MR 17, 255.

3. P. J. DAVIS, Interpolation and Approximation, Blaisdell, Waltham, Mass., 1963. MR 28 \# 393.

4. E. T. Copson, Theory of Functions of a Complex Variable, Oxford Univ. Press, Oxford, 1935.

5. M. M. ChAWLA \& M. K. JAIN, "Error estimates for Gauss quadrature formulas for analytic functions," Math. Comp., v. 22, 1968, pp. 82-90. MR 36 \#6142.

6. M. M. ChAWLA, "Asymptotic estimates for the error of the Gauss-Legendre quadrature formula," Comput. J., v. 11, 1968, pp. 339-340. MR 38 \#5389.

7. F. STENGER, "Bounds on the error of Gauss-type quadratures," Numer. Math., v. 8, 1966, pp. 150-160. MR 33 \#5120. 